\title{
REMOVING ISOLATED ZEROES BY HOMOTOPY
}

\author{
ADAM COFFMAN - JIŘÍ LEBL
}

\begin{abstract}
Suppose that the inverse image of the zero vector by a continuous map $f: \mathbb{R}^{n} \rightarrow \mathbb{R}^{q}$ has an isolated point $P$. The existence of a continuous map $g$ which approximates $f$ but is nonvanishing near $P$ is equivalent to a topological property we call "locally inessential," generalizing the notion of index zero for vector fields, the $q=n$ case. We consider the problem of constructing such an approximation $g$ and a continuous homotopy $F(x, t)$ from $f$ to $g$ through locally nonvanishing maps. If $f$ is a semialgebraic map, then there exists $F$ also semialgebraic. If $q=2$ and $f$ is real analytic with a locally inessential zero, then there exists a Hölder continuous homotopy $F(x, t)$ which, for $(x, t) \neq(P, 0)$, is real analytic and nonvanishing. The existence of a smooth homotopy, given a smooth map $f$, is stated as an open question.
\end{abstract}

\section{INTRODUCTION}

For a continuous vector field on a manifold, it is well-known that an isolated zero can be removed by a small, local perturbation if and only if that zero has an "index" equal to 0 . That is, for a vector field $\mathbf{f}$ vanishing with index 0 at $\vec{p}$, and any small neighborhood of $\vec{p}$, there is another vector field $\mathbf{g}$ agreeing with $\mathbf{f}$ outside that neighborhood, and arbitrarily $\mathcal{C}^{0}$-close to $\mathbf{f}$ but nonvanishing inside it. In fact, the zero is removable in the following stronger, but less well-known, sense $\left(\mathrm{D}_{3}\right)$ : not only is there such a perturbation $\mathbf{g}$, there is a continuous homotopy $F(\vec{x}, t)$ from $\mathbf{f}$ to $\mathbf{g}$ such that $F(\vec{x}, t)$ is nonvanishing for $\vec{x}$ near $\vec{p}$ and $t>0$. So, the isolated zero can be removed instantaneously.

We consider the generalization of this phenomenon to other dimensions: locally, maps $\mathbf{f}$ : $\mathbb{R}^{n} \rightarrow \mathbb{R}^{q}$, but our primary interest is in the regularity of the homotopy $F$. One version of our Main Question 4.3 asks: given that $\mathbf{f}$ is smooth, does there exist a smooth homotopy $F$ that instantly removes an isolated zero, assuming only that there is no topological obstruction to a nonvanishing approximation? This remains open; as remarked by $\left[\mathrm{D}_{2}\right], \mathrm{D}_{3}$, just $\mathcal{C}^{1}$ regularity for $F$ seems to be a difficult question even under strong assumptions about $\mathbf{f}$. Our main results consider cases where $\mathbf{f}$ is semialgebraic (Theorem 4.1) or real analytic (Theorem [5.1).

When the target dimension $q$ is not equal to $n$, isolated zeros are no longer a generic phenomenon, but in Section 3, we show that with a natural generalization of the notion of "index zero" (Definition 3.2), the analogous nonvanishing approximation property holds. Instead of considering $\mathbf{f}$ as a vector field, another way to visualize an isolated zero of $\mathbf{f}: \mathbb{R}^{n} \rightarrow \mathbb{R}^{q}$ is to consider the zero sets of its components $\left(f_{1}(\vec{x}), \ldots, f_{q}(\vec{x})\right)$. For $q$ suitably smooth and generic functions, each component vanishes on some hypersurface, and for $q \leq n$, the intersection of $q$ hypersurfaces in general position is expected to be a set with dimension $n-q$. So for $q<n$, an isolated point in the intersection indicates that the hypersurfaces are not in general position, but our question is about the persistence of the isolated zero: is there some perturbation so that the isolated point of intersection disappears, or do the $q$ hypersurfaces continue to have a

2010 Mathematics Subject Classification. Primary 57R45; Secondary 14P10, 31B25, 35J25, 41A29, 57R25, $58 \mathrm{~K} 25,58 \mathrm{~K} 45,58 \mathrm{~K} 60$.

Key words and phrases. Isolated zero; semialgebraic map; singularities of differentiable mappings.

The second author was in part supported by NSF grant DMS-1362337. 
non-empty, but not necessarily isolated, intersection after any perturbation? In the case $n=3$, $q=2$ (of special interest for applications such as computer graphics), can two real implicit surfaces $\left\{f_{1}=0\right\},\left\{f_{2}=0\right\}$, meeting at just one point (e.g., two cones sharing a vertex; for another example, see Section (6), always be made disjoint by small changes in $f_{1}$ and $f_{2}$ ? The answer is yes, and "small changes" can be interpreted as either a local $\mathcal{C}^{0}$ approximation, or a continuous family of such approximations (the homotopy $F$ ).

Our first approach to the construction of $F$ is to start with the local approximation; the results in Section 3 generalize well-known facts about the vector field case $(q=n)$. Then in Section 4 we construct the continuous homotopy; the $q=n$ case was considered by $\mathrm{D}_{3}$; our construction for any $q$ in Theorem 4.1 is explicit enough so that the homotopy $F$ is semialgebraic if $\mathbf{f}$ is.

The second approach, in Section 5, considers the case where $q=2$ and $\mathbf{f}$ is real analytic; the construction of Theorem 5.1 uses PDE methods (only the classical Dirichlet problem, with Hölder estimates up to the boundary) to construct a continuous nonvanishing homotopy $F$ which is real analytic except at the point $(\vec{p}, 0)$, near which it satisfies an inequality of the form $\|F(\vec{x}, t)\| \leq C\|(\vec{x}-\vec{p}, t)\|$. Either better regularity or a counterexample would be interesting: is there some polynomial map with an isolated zero that can be removed by a semialgebraic homotopy but not by a $\mathcal{C}^{1}$ homotopy?

Time-dependent vector fields are of obvious importance in applications, and the behavior of their zeros remains a topic of current interest ([NNPV]). We further remark that the global problem of finding a homotopy from a map $\mathbf{f}$ to another map with fewer zeros has been considered in algebraic topology ([BFGJ], $[\mathrm{F}]$ ). The topic of "root theory" is a special case of the coincidence problem of finding homotopies from two maps $\mathbf{f}$ and $\mathbf{g}$ at time 0 to two other maps at time 1 with disjoint images (or with a minimal number of points of intersection). Our results in Sections 4 and 5 are different, in that we want to find a homotopy where the image of $\mathbf{f}$ becomes disjoint from $\{\mathbf{0}\}$ for all $t>0$.

Finally, we mention that our interest in this topic started with an analogue in CR geometry [E] considers a real 3-manifold embedded in $\mathbb{C}^{3}$ with an isolated complex tangent, and describes a local topological obstruction to the existence of a $\mathcal{C}^{1}$ homotopy to a totally real embedding.

\section{NotATiOn}

Fix a positive integer $n$. We are interested in maps $\mathbf{f}$ from an $n$-manifold to another manifold, where the inverse image of a point $O$ contains an isolated point. Our analysis is local, not global, so we can consider the target manifold to be $\mathbb{R}^{q}$ for some $q$ and the point $O$ to be the zero vector $\mathbf{0}$; then an isolated point of $\mathbf{f}^{-1}(\{O\})$ is called an isolated zero of $\mathbf{f}$ (Definition 2.3). We consider the domain of $\mathbf{f}$ and its perturbations to be a neighborhood $\Omega$ in $\mathbb{R}^{n}$ rather than a more general $n$-manifold.

Notation 2.1. For $\vec{c} \in \mathbb{R}^{n}$ and $R>0$, the following notation is used for the standard Euclidean balls and spheres with center $\vec{c}$ and radius $R$ :

$$
\begin{aligned}
B^{n}(\vec{c}, R) & =\{\vec{x}:\|\vec{x}-\vec{c}\|<R\}=\text { the open ball } \\
\bar{B}^{n}(\vec{c}, R) & =\{\vec{x}:\|\vec{x}-\vec{c}\| \leq R\}=\text { the closed ball } \\
B_{*}^{n}(\vec{c}, R) & =\{\vec{x}: 0<\|\vec{x}-\vec{c}\|<R\}=\text { the punctured open ball } \\
\bar{B}_{*}^{n}(\vec{c}, R) & =\{\vec{x}: 0<\|\vec{x}-\vec{c}\| \leq R\}=\text { the punctured closed ball } \\
S^{n-1}(\vec{c}, R) & =\{\vec{x}:\|\vec{x}-\vec{c}\|=R\}=\text { the boundary sphere }
\end{aligned}
$$


Similarly, for $\mathbb{R}^{n+1}$ with coordinates $(\vec{x}, t)$, denote the upper half-ball and upper hemisphere:

$$
\begin{aligned}
B_{+}^{n+1}((\vec{c}, \tau), R) & =\{(\vec{x}, t):\|(\vec{x}, t)-(\vec{c}, \tau)\|<R, t>\tau\} \\
S_{+}^{n}((\vec{c}, \tau), R) & =\{(\vec{x}, t):\|(\vec{x}, t)-(\vec{c}, \tau)\|=R, t>\tau\}
\end{aligned}
$$

The usual unit sphere $S^{q-1}(\overrightarrow{0}, 1)$ in $\mathbb{R}^{q}$ will be abbreviated $S^{q-1}$. The restriction of a map $\mathbf{f}: \Omega \rightarrow \mathbb{R}^{q}$ to some sphere $S^{n-1}(\vec{c}, r) \subseteq \Omega$ will be denoted $\left.\mathbf{f}\right|_{S}$.

Notation 2.2. For a map $\mathbf{f}: \Omega \rightarrow \mathbb{R}^{q}$ with components $\mathbf{f}(\vec{x})=\left(f_{1}(\vec{x}), \ldots, f_{q}(\vec{x})\right)$, denote the locus:

$$
V(\mathbf{f})=V\left(f_{1}, \ldots, f_{q}\right)=\left\{\vec{x} \in \Omega: f_{1}(\vec{x})=0, \ldots, f_{q}(\vec{x})=0\right\}=\mathbf{f}^{-1}(\{\mathbf{0}\}) .
$$

Definition 2.3. For a function $\mathbf{f}: \Omega \rightarrow \mathbb{R}^{q}$ and a point $\vec{p} \in \Omega$ such that $\mathbf{f}(\vec{p})=\mathbf{0}, \vec{p}$ is an isolated zero of $\mathbf{f}$ means that there exists some $R>0$ so that $B^{n}(\vec{p}, R) \subseteq \Omega$ and:

$$
V(\mathbf{f}) \cap B^{n}(\vec{p}, R)=\{\vec{p}\} .
$$

Definition 2.4. A subset $\Sigma \subseteq \mathbb{R}^{N}$ is a semialgebraic set means that $\Sigma$ is a finite union of sets of the form

$$
\left\{\vec{x} \in \mathbb{R}^{N}: P(\vec{x})=0, Q_{1}(\vec{x})>0, \ldots, Q_{j}(\vec{x})>0\right\},
$$

where each of the functions $P, Q_{1}, \ldots, Q_{j}$ is a polynomial $\mathbb{R}^{N} \rightarrow \mathbb{R}^{1}$. For an open domain $\Omega \subseteq \mathbb{R}^{n}$ as above, a function $\mathbf{f}: \Omega \rightarrow \mathbb{R}^{q}$ is a semialgebraic map means that the graph of $\mathbf{f}$ is a semialgebraic set in $\mathbb{R}^{n+q}$.

Remark 2.5. Some references require that semialgebraic maps are also continuous; it is more convenient for us to instead allow discontinuous maps and explicitly mention continuity when needed.

Proposition 2.6. A linear projection of a semialgebraic set is a semialgebraic set. The scalar valued semialgebraic maps $f: \mathbb{R}^{n} \rightarrow \mathbb{R}^{1}$ form a ring. A map $\mathbf{f}: \mathbb{R}^{n} \rightarrow \mathbb{R}^{q}$ is semialgebraic if and only if its components, $\mathbf{f}=\left(f_{1}, \ldots, f_{q}\right)$, are semialgebraic $f_{j}: \mathbb{R}^{n} \rightarrow \mathbb{R}^{1}$. The composite of semialgebraic maps is semialgebraic.

Remark 2.7. The notions of Definition 2.4 and the claims of Proposition 2.6 are well-known (we refer to $\mathrm{BCR}]$ ). For an open domain $\Omega$, it follows from Definition 2.4 and the projection property that if $\mathbf{f}: \Omega \rightarrow \mathbb{R}^{q}$ is a semialgebraic map, then $\Omega$ must be an open semialgebraic set.

\section{NONVANISHING APPROXIMATION}

Theorem 3.6 and its Corollaries are about approximating a map with an isolated zero by a nonvanishing map. The case $q=n$ is the well-known situation of a vector field with index zero, for which [ST, $\mathrm{A}_{1}, \mathrm{~A}_{2}$, prove results similar to Theorem 3.6 and additionally give estimates for derivatives (see also $[\mathrm{PP}]$ ). For $q \leq n$, the connection between zero sets of perturbations and homotopy classes of maps of spheres was used by $\mathrm{D}_{1}$ to study the stability of non-isolated zero sets, rather than the removability of isolated zeros.

Definition 3.2 generalizes the notion of "index zero" to other dimensions. For this Section, fix a positive integer $q$.

Lemma 3.1. For a continuous map $\mathbf{f}: \Omega \rightarrow \mathbb{R}^{q}$, and an isolated zero $\vec{p} \in B^{n}(\vec{p}, R)$ as in Definition 2.3, the following are equivalent:

(i) There exists $r \in(0, R)$ such that the restriction of $\mathbf{f}$ to the domain $S^{n-1}(\vec{p}, r)$ and target $\mathbb{R}^{q} \backslash\{\mathbf{0}\}$ is null-homotopic; 
(ii) For any $r \in(0, R)$, the restriction of $\mathbf{f}$ to the domain $S^{n-1}(\vec{p}, r)$ and target $\mathbb{R}^{q} \backslash\{\mathbf{0}\}$ is null-homotopic;

(iii) For any $\epsilon>0$ there is some $\delta$ so that $0<\delta \leq R$ and for any $r \in(0, \delta)$, the restriction of f to the domain $S^{n-1}(\vec{p}, r)$ and target $B_{*}^{q}(\mathbf{0}, \epsilon)$ is null-homotopic;

(iv) For any $\epsilon>0$ there is some $\delta$ so that $0<\delta \leq R$ and for any $r \in(0, \delta)$, the restriction of $\mathbf{f}$ to the domain $B_{*}^{n}(\vec{p}, r)$ and target $B_{*}^{q}(\mathbf{0}, \epsilon)$ is null-homotopic.

Proof. The implication (iv) $\Longrightarrow$ (i) is obvious. The statement of Property (i) is the most easily checked, while the statements of Properties (iii) and (iv) are local both in the domain and the target, and could be adapted for isolated roots of a map from one manifold to another.

To show that $(\mathrm{i}) \Longrightarrow\left(\right.$ ii), let $r_{0}$ be the radius from Property (i), so that there exists a homotopy $\Phi: S^{n-1}\left(\vec{p}, r_{0}\right) \times[0,1] \rightarrow \mathbb{R}^{q} \backslash\{\mathbf{0}\}$ from $\Phi(\vec{x}, 0)=\left.\mathbf{f}\right|_{S}(\vec{x})$ to a constant map $\Phi(\vec{x}, 1) \equiv \mathbf{c}$ in $\mathbb{R}^{q} \backslash\{\mathbf{0}\}$. For any $r \in(0, R)$, define $\Phi_{r}: S^{n-1}(\vec{p}, r) \times[0,1] \rightarrow \mathbb{R}^{q} \backslash\{\mathbf{0}\}$ by the formula

$$
\Phi_{r}(\vec{x}, t)=\left\{\begin{array}{cc}
\mathbf{f}\left(\vec{p}+\left(1+2\left(\frac{r_{0}}{r}-1\right) t\right) \cdot(\vec{x}-\vec{p})\right) & 0 \leq t \leq \frac{1}{2} \\
\Phi\left(\vec{p}+\frac{r_{0}}{r} \cdot(\vec{x}-\vec{p}), 2 t-1\right) & \frac{1}{2} \leq t \leq 1
\end{array}\right\} .
$$

Then $\Phi_{r}$ is a continuous homotopy from $\Phi_{r}(\vec{x}, 0)=\left.\mathbf{f}\right|_{S}(\vec{x})$ to the constant map $\Phi_{r}(\vec{x}, 1) \equiv \mathbf{c}$ in $\mathbb{R}^{q} \backslash\{\mathbf{0}\}$.

Assuming (ii), for any $\epsilon>0$ there is, by the continuity of $\mathbf{f}$, some $\delta, 0<\delta \leq R$, such that if $\|\vec{x}-\vec{p}\|<\delta$ then $\|\mathbf{f}(\vec{x})-\mathbf{f}(\vec{p})\|<\epsilon$. For any $r \in(0, \delta)$, the image $\mathbf{f}\left(S^{n-1}(\vec{p}, r)\right)$ is contained in $B_{*}^{q}(\mathbf{0}, \epsilon)$. By Property (ii), $\left.\mathbf{f}\right|_{S}$ is homotopic in $\mathbb{R}^{q} \backslash\{\mathbf{0}\}$ to a constant map; the claim of Property (iii) is just that it is also homotopic in $B_{*}^{q}(\mathbf{0}, \epsilon)$ to a (possibly different) constant map.

Let $\Phi_{r}: S^{n-1}(\vec{p}, r) \times[0,1] \rightarrow \mathbb{R}^{q} \backslash\{\mathbf{0}\}$ be the homotopy from Property (ii), so that $\Phi_{r}(\vec{x}, 0)=$ $\left.\mathbf{f}\right|_{S}(\vec{x})$ and $\Phi_{r}(\vec{x}, 1) \equiv \mathbf{c}_{r} \in \mathbb{R}^{q} \backslash\{\mathbf{0}\}$. By the continuity of $\Phi_{r}$ and the compactness of $S^{n-1}(\vec{p}, r)$, there is some $\delta_{2}, 0<\delta_{2} \leq 1$, so that the image $\Phi_{r}\left(S^{n-1}(\vec{p}, r) \times\left[0, \delta_{2}\right]\right)$ is contained in $B_{*}^{q}(\mathbf{0}, \epsilon)$. Also, $\Phi_{r}$ achieves a maximum magnitude $M=\max \left\{\left\|\Phi_{r}(\vec{x}, t)\right\|:(\vec{x}, t) \in S^{n-1}(\vec{p}, r) \times[0,1]\right\}>0$. If $\delta_{2}=1$ or $M<\epsilon$, Property (iii) already holds; otherwise, for $0<\delta_{2}<1$ and $M \geq \epsilon$, define the following weakly decreasing, continuous function $\gamma:[0,1] \rightarrow(0,1]$ :

$$
\gamma(t)=\left\{\begin{array}{cc}
\frac{\frac{\epsilon}{2 M}-1}{\delta_{2}} t+1 & 0 \leq t \leq \delta_{2} \\
\frac{\epsilon}{2 M} & \delta_{2} \leq t \leq 1
\end{array}\right\}
$$

Then $\Psi(\vec{x}, t)=\gamma(t) \cdot \Phi_{r}(\vec{x}, t)$ is a continuous homotopy from $\Psi(\vec{x}, 0)=1 \cdot \Phi_{r}(\vec{x}, 0)=\mathbf{f}(\vec{x})$ to the constant map $\Psi(\vec{x}, 1)=\frac{\epsilon}{2 M} \cdot \mathbf{c}_{r} \in B_{*}^{q}(\mathbf{0}, \epsilon)$. For $0 \leq t \leq \delta_{2},\|\Psi(\vec{x}, t)\|=\gamma(t)\left\|\Phi_{r}(\vec{x}, t)\right\|<1 \cdot \epsilon$, and for $\delta_{2} \leq t \leq 1,\|\Psi(\vec{x}, t)\|=\frac{\epsilon}{2 M}\left\|\Phi_{r}(\vec{x}, t)\right\| \leq \frac{\epsilon}{2}$, which establishes Property (iii).

Assuming (iii), for any $\epsilon>0$ there is, by the continuity of $\mathbf{f}$, some $\delta_{1}, 0<\delta_{1} \leq R$, such that if $\|\vec{x}-\vec{p}\|<\delta_{1}$ then $\|\mathbf{f}(\vec{x})-\mathbf{f}(\vec{p})\|<\epsilon$. There is, from Property (iii), some $\delta_{2}>0$ corresponding to the same $\epsilon$; the claimed $\delta$ will be $\min \left\{\delta_{1}, \delta_{2}\right\}$. Let $r$ be any radius in $(0, \delta)$, and then there is a homotopy $\Psi_{r}: S^{n-1}(\vec{p}, r) \times[0,1] \rightarrow B_{*}^{q}(\mathbf{0}, \epsilon)$ from $\left.\mathbf{f}\right|_{S}$ to a constant $\mathbf{c}$ in $B_{*}^{q}(\mathbf{0}, \epsilon)$. The map $\Theta: B_{*}^{n}(\vec{p}, r) \times[0,1] \rightarrow B_{*}^{q}(\mathbf{0}, \epsilon)$, defined by the following formula, is a homotopy in $B_{*}^{q}(\mathbf{0}, \epsilon)$ as claimed in Property (iv):

$$
\Theta(\vec{x}, t)=\left\{\begin{array}{cc}
\mathbf{f}\left(\vec{p}+\left(\frac{2(r-\|\vec{x}-\vec{p}\|) t+\|\vec{x}-\vec{p}\|}{\|\vec{x}-\vec{p}\|}\right) \cdot(\vec{x}-\vec{p})\right) & 0 \leq t \leq \frac{1}{2} \\
\Psi_{r}\left(\vec{p}+\frac{r}{\|\vec{x}-\vec{p}\|} \cdot(\vec{x}-\vec{p}), 2 t-1\right) & \frac{1}{2} \leq t \leq 1
\end{array}\right\} .
$$

Definition 3.2. For a continuous map $\mathbf{f}: \Omega \rightarrow \mathbb{R}^{q}$, a point $\vec{p}$ such that $\mathbf{f}(\vec{p})=\mathbf{0}$ is a locally inessential zero of $\mathbf{f}$ means: $\vec{p}$ is an isolated zero and any of the equivalent properties of Lemma 3.1 is satisfied. 
Remark 3.3. For the case $q=n$, the above notion is exactly that the isolated zero of $\mathbf{f}$ has Poincaré-Hopf index 0. The new term "locally inessential zero" could be replaced by just using "isolated zero with index 0" more generally for any $q$, $n$, but such usage would raise the unrelated (for us) question of whether or how one could define a non-zero index for $q \neq n$.

Lemma 3.4. If $n$ and $q$ satisfy

$$
\pi_{n-1}\left(S^{q-1}\right) \cong\{0\}
$$

then any isolated zero of any continuous map $\mathbf{f}: \Omega \rightarrow \mathbb{R}^{q}$ is a locally inessential zero.

Proof. For $\vec{p}$ and $R>0$ as in Definition 2.3, let $r$ be any radius in $(0, R)$; we will check Property (i) from Lemma 3.1. The space $\mathbb{R}^{q} \backslash\{\mathbf{0}\}$ is homeomorphic to the product $S^{q-1} \times(0,1)$, so its $(n-1)^{t h}$ homotopy group is the same as that of $S^{q-1}$, and trivial by hypothesis. Any continuous map from an $(n-1)$-sphere to $\mathbb{R}^{q} \backslash\{\mathbf{0}\}$, including the restriction $\left.\mathbf{f}\right|_{S}$ from Property (i), is homotopic to a constant map.

Remark 3.5. The hypothesis on $n$ and $q$ in Lemma 3.4 is satisfied for $q=2$ and $n \geq 3$, or for any pair where $n<q$.

Theorem 3.6. Let $\mathbf{f}: \Omega \rightarrow \mathbb{R}^{q}$ be a continuous map with an isolated zero at $\vec{p}$ and $R>0$ as in Definition 2.3. The following are equivalent:

- $\vec{p}$ is a locally inessential zero of $\mathbf{f}$;

- For any $\epsilon>0$ and any $\rho$ with $0<\rho \leq R$, there exists a continuous map $\mathbf{g}: \Omega \rightarrow \mathbb{R}^{q}$ such that:

$$
\begin{aligned}
\vec{x} \in \Omega \backslash B^{n}(\vec{p}, \rho) \Longrightarrow \mathbf{g}(\vec{x}) & =\mathbf{f}(\vec{x}), \quad \text { and } \\
\vec{x} \in \Omega \Longrightarrow\|\mathbf{g}(\vec{x})-\mathbf{f}(\vec{x})\| & <\epsilon, \text { and } \\
V(\mathbf{g}) \cap B^{n}(\vec{p}, R) & =\varnothing .
\end{aligned}
$$

Proof. First, assume that $\vec{p}$ is a locally inessential zero. Given $\epsilon>0$, there is some $\delta_{1}>0$ corresponding to $\frac{\epsilon}{2}$ in Property (iii) from Lemma 3.1. Let $\delta=\min \left\{\delta_{1}, \rho\right\}$, and denote by $\left.\mathbf{f}\right|_{S}$ the restriction of $\mathbf{f}$ to the domain $S^{n-1}\left(\vec{p}, \frac{\delta}{2}\right)$ and target $B_{*}^{q}\left(\mathbf{0}, \frac{\epsilon}{2}\right)$.

By Property (iii) from Lemma 3.1, there exists a homotopy

$$
\varphi: S^{n-1}\left(\vec{p}, \frac{\delta}{2}\right) \times[0,1] \rightarrow B_{*}^{q}\left(\mathbf{0}, \frac{\epsilon}{2}\right)
$$

from $\varphi(\vec{x}, 0)=\left.\mathbf{f}\right|_{S}(\vec{x})$ to a constant map $\varphi(\vec{x}, 1) \equiv \mathbf{c}$ in $B_{*}^{q}\left(\mathbf{0}, \frac{\epsilon}{2}\right)$. By compactness, $\varphi$ achieves some minimum magnitude $0<m \leq\|\varphi(\vec{x}, t)\|<\frac{\epsilon}{2}$. Now $\left.\mathbf{f}\right|_{S}$ extends to a continuous $\tilde{\mathbf{f}}$ : $\bar{B}^{n}\left(\vec{p}, \frac{\delta}{2}\right) \rightarrow B_{*}^{q}\left(\mathbf{0}, \frac{\epsilon}{2}\right)$. In the interest of giving explicit formulas when we can, the extension constructed in $[\underline{S}] \S 1.3$ is defined by:

$$
\tilde{\mathbf{f}}(\vec{x})=\left\{\begin{array}{cc}
\mathbf{c} & 0 \leq\|\vec{x}-\vec{p}\| \leq \frac{\delta}{4} \\
\varphi\left(\vec{p}+\frac{\delta}{2\|\vec{x}-\vec{p}\|}(\vec{x}-\vec{p}), 2-\frac{4}{\delta}\|\vec{x}-\vec{p}\|\right) & \frac{\delta}{4} \leq\|\vec{x}-\vec{p}\| \leq \frac{\delta}{2}
\end{array}\right\},
$$

which is continuous because its two pieces are continuous on closed sets and agree on their intersection.

By construction, for all $\vec{x} \in \bar{B}^{n}\left(\vec{p}, \frac{\delta}{2}\right), 0<m \leq\|\tilde{\mathbf{f}}(\vec{x})\|<\frac{\epsilon}{2}$, and for all $\vec{x} \in S^{n-1}\left(\vec{p}, \frac{\delta}{2}\right)$, $\tilde{\mathbf{f}}(\vec{x})=\left.\mathbf{f}\right|_{S}(\vec{x})=\mathbf{f}(\vec{x})$. Define $\mathbf{g}: \Omega \rightarrow \mathbb{R}^{q}$ by $\mathbf{g}(\vec{x})=\mathbf{f}(\vec{x})$ for $\|\vec{x}-\vec{p}\| \geq \frac{\delta}{2}$, and $\mathbf{g}(\vec{x})=\tilde{\mathbf{f}}(\vec{x})$ for $\|\vec{x}-\vec{p}\| \leq \frac{\delta}{2}$; then $\mathbf{g}$ is continuous on $\Omega$, and satisfies (11) and (3) as claimed. Further, for any $\vec{x} \in \Omega$, either $\|\mathbf{g}(\vec{x})-\mathbf{f}(\vec{x})\|=0$ or

$$
\|\mathbf{g}(\vec{x})-\mathbf{f}(\vec{x})\|=\|\tilde{\mathbf{f}}(\vec{x})-\mathbf{f}(\vec{x})\| \leq\|\tilde{\mathbf{f}}(\vec{x})\|+\|\mathbf{f}(\vec{x})\|<\frac{\epsilon}{2}+\frac{\epsilon}{2}=\epsilon .
$$


Conversely, for any $\epsilon>0$, there is some $\delta, 0<\delta \leq R$, so that if $\|\vec{x}-\vec{p}\|<\delta$ then $\|\mathbf{f}(\vec{x})\|<\frac{\epsilon}{2}$. Let $\rho=\frac{\delta}{2}$, so there is a continuous $\mathbf{g}$ with $\|\mathbf{g}(\vec{x})-\mathbf{f}(\vec{x})\|<\frac{\epsilon}{2}$ for all $\vec{x} \in \Omega$, and $\mathbf{g}(\vec{x})=\mathbf{f}(\vec{x})$ for all $\vec{x} \in \Omega \backslash B^{n}(\vec{p}, \rho)$. Let $\left.\mathbf{f}\right|_{S}$ denote the restriction of $\mathbf{f}$ to the domain $S^{n-1}(\vec{p}, \rho)$ and target $B_{*}^{q}(\mathbf{0}, \epsilon)$. Define $\Psi_{\rho}: S^{n-1}(\vec{p}, \rho) \times[0,1] \rightarrow \mathbb{R}^{q} \backslash\{\mathbf{0}\}$ by

$$
\Psi_{\rho}(\vec{x}, t)=\mathbf{g}(\vec{p}+(1-t)(\vec{x}-\vec{p})) .
$$

So, $\Psi_{\rho}(\vec{x}, 0)=\left.\mathbf{g}\right|_{S}(\vec{x})=\left.\mathbf{f}\right|_{S}(\vec{x}), \Psi_{\rho}(\vec{x}, 1) \equiv \mathbf{g}(\vec{p})$, and

$$
\begin{aligned}
\left\|\Psi_{\rho}(\vec{x}, t)\right\| & =\|\mathbf{g}(\vec{p}+(1-t)(\vec{x}-\vec{p}))\| \\
& \leq\|\mathbf{g}(\vec{p}+(1-t)(\vec{x}-\vec{p}))-\mathbf{f}(\vec{p}+(1-t)(\vec{x}-\vec{p}))\|+\|\mathbf{f}(\vec{p}+(1-t)(\vec{x}-\vec{p}))\| \\
& <\frac{\epsilon}{2}+\frac{\epsilon}{2}=\epsilon,
\end{aligned}
$$

so Property (iii) from Lemma 3.1 holds.

Corollary 3.7. Let $q, \mathbf{f}, \vec{p}$, and $R$ be as in Theorem 3.6. If $\vec{p}$ is a locally inessential zero of $\mathbf{f}$, and, additionally, $\mathbf{f}$ is smooth, then there exists $\mathbf{g}$ as in Theorem 3.6, and which is also smooth.

Proof. Given $\epsilon>0$, apply the construction of Theorem 3.6 to $\mathbf{f}$ to get a continuous $\mathbf{g}_{0}$ satisfying (2) with $\frac{\epsilon}{2}$, and a corresponding $\delta>0$. By construction, for $\vec{x} \in \bar{B}^{n}\left(\vec{p}, \frac{\delta}{2}\right), 0<m \leq\left\|\mathbf{g}_{0}(\vec{x})\right\|<\frac{\epsilon}{4}$, and if $\mathbf{f}$ is smooth $\left(\mathcal{C}^{\infty}\right)$ on $\Omega$, then $\mathbf{g}_{0}$ is equal to a smooth map on the closed set $\Omega \backslash B^{n}\left(\vec{p}, \frac{\delta}{2}\right)$. By the Whitney Approximation Theorem ( $[\mathrm{L}]$ Ch. 6), there exists a smooth $\mathrm{g}: \Omega \rightarrow \mathbb{R}^{q}$ such that $\mathbf{g} \equiv \mathbf{g}_{0} \equiv \mathbf{f}$ on $\Omega \backslash B^{n}\left(\vec{p}, \frac{\delta}{2}\right)$, so (1) is satisfied, and for all $\vec{x} \in \Omega$,

$$
\left\|\mathbf{g}(\vec{x})-\mathbf{g}_{0}(\vec{x})\right\|<\frac{m}{2}<\frac{\epsilon}{8} .
$$

$\mathbf{g}$ is then close to $\mathbf{f}$ (satisfying (2) ):

$$
\|\mathbf{g}(\vec{x})-\mathbf{f}(\vec{x})\| \leq\left\|\mathbf{g}(\vec{x})-\mathbf{g}_{0}(\vec{x})\right\|+\left\|\mathbf{g}_{0}(\vec{x})-\mathbf{f}(\vec{x})\right\|<\frac{\epsilon}{8}+\frac{\epsilon}{2}<\epsilon,
$$

and has the claimed nonvanishing property (3):

$$
\vec{x} \in B^{n}\left(\vec{p}, \frac{\delta}{2}\right) \Longrightarrow\|\mathbf{g}(\vec{x})\| \geq\left\|\mathbf{g}_{0}(\vec{x})\right\|-\left\|\mathbf{g}(\vec{x})-\mathbf{g}_{0}(\vec{x})\right\|>m-\frac{m}{2} .
$$

Corollary 3.8. Let $q, \mathbf{f}, \vec{p}$, and $R$ be as in Theorem 3.6. If $\vec{p}$ is an inessential zero of $\mathbf{f}$, and, additionally, $\mathbf{f}$ is a continuous, semialgebraic map, then there exists $\mathbf{g}$ as in Theorem 3.6, and which is a continuous, semialgebraic map.

Proof. Given $\epsilon>0$, apply the construction of Theorem 3.6 to $\mathbf{f}$ to get a continuous $\mathbf{g}_{0}$ satisfying (2) with $\frac{\epsilon}{2}$, and corresponding $\delta>0, m>0$. By construction, for $\vec{x} \in \bar{B}^{n}\left(\vec{p}, \frac{\delta}{2}\right),\|\mathbf{f}(\vec{x})\|<\frac{\epsilon}{4}$, and $m \leq\left\|\mathbf{g}_{0}(\vec{x})\right\|<\frac{\epsilon}{4}$. Also, $\mathbf{f}-\mathbf{g}_{0}$ is continuous on $\Omega$, and $\equiv \mathbf{0}$ on $S^{n-1}\left(\vec{p}, \frac{\delta}{2}\right)$, so by compactness, there is some $\delta_{1}$ so that $0<\delta_{1}<\frac{\delta}{2}$ and if $\delta_{1} \leq\|\vec{x}-\vec{p}\| \leq \frac{\delta}{2}$, then $\left\|\mathbf{f}(\vec{x})-\mathbf{g}_{0}(\vec{x})\right\|<\frac{m}{2}$.

Apply the Weierstrass Approximation Theorem to $\mathbf{g}_{0}$ on the compact set $\bar{B}^{n}\left(\vec{p}, \frac{\delta}{2}\right)$ to get polynomials $h_{1}, \ldots, h_{q}$ so that for $\vec{x} \in \bar{B}^{n}\left(\vec{p}, \frac{\delta}{2}\right)$, the map $\mathbf{h}=\left(h_{1}, \ldots, h_{q}\right)$ satisfies

$$
\left\|\mathbf{h}(\vec{x})-\mathbf{g}_{0}(\vec{x})\right\|<\frac{m}{2}<\frac{\epsilon}{8}
$$


Let $\chi: \mathbb{R}^{1} \rightarrow \mathbb{R}^{1}$ be a continuous, piecewise linear, weakly decreasing cutoff function:

$$
\chi(s)=\left\{\begin{array}{cl}
1 & s \leq \delta_{1} \\
\frac{s-\frac{\delta}{2}}{\delta_{1}-\frac{\delta}{2}} & \delta_{1} \leq s \leq \frac{\delta}{2} \\
0 & s \geq \frac{\delta}{2}
\end{array}\right\} .
$$

Define $\mathbf{g}: \Omega \rightarrow \mathbb{R}^{q}$ by:

$$
\mathbf{g}(\vec{x})=\chi(\|\vec{x}-\vec{p}\|) \cdot \mathbf{h}(\vec{x})+(1-\chi(\|\vec{x}-\vec{p}\|)) \cdot \mathbf{f}(\vec{x}) .
$$

By Proposition 2.6, $\mathrm{g}$ is semialgebraic, and by construction, $\mathrm{g}$ is continuous and satisfies (1).

$\mathbf{g}$ is close to $\mathbf{f}$ (satisfying (2)) - either $\mathbf{g}(\vec{x})=\mathbf{f}(\vec{x})$, or for $\vec{x} \in B^{n}\left(\vec{p}, \frac{\delta}{2}\right)$ :

$$
\begin{aligned}
\|\mathbf{g}(\vec{x})-\mathbf{f}(\vec{x})\| & =\|\chi \cdot(\mathbf{h}(\vec{x})-\mathbf{f}(\vec{x}))\| \\
& \left.\leq \chi \cdot\left(\left\|\mathbf{h}(\vec{x})-\mathbf{g}_{0}(\vec{x})\right\|+\| \mathbf{g}_{0}(\vec{x})-\mathbf{f}(\vec{x})\right) \|\right) \\
& <\frac{\epsilon}{8}+\frac{\epsilon}{2} .
\end{aligned}
$$

g has the claimed nonvanishing property (3) ; for $\vec{x} \in B^{n}\left(\vec{p}, \delta_{1}\right)$ :

$$
\|\mathbf{g}(\vec{x})\|=\|\mathbf{h}(\vec{x})\| \geq\left\|\mathbf{g}_{0}(\vec{x})\right\|-\left\|\mathbf{h}(\vec{x})-\mathbf{g}_{0}(\vec{x})\right\|>m-\frac{m}{2},
$$

and for $\vec{x} \in B^{n}\left(\vec{p}, \frac{\delta}{2}\right) \backslash B^{n}\left(\vec{p}, \delta_{1}\right)$ :

$$
\begin{aligned}
\|\mathbf{g}(\vec{x})\| & =\|\chi \cdot \mathbf{h}(\vec{x})+(1-\chi) \cdot \mathbf{f}(\vec{x})\| \\
& =\left\|\mathbf{g}_{0}(\vec{x})+\chi \cdot\left(\mathbf{h}(\vec{x})-\mathbf{g}_{0}(\vec{x})\right)+(1-\chi) \cdot\left(\mathbf{f}(\vec{x})-\mathbf{g}_{0}(\vec{x})\right)\right\| \\
& \geq\left\|\mathbf{g}_{0}(\vec{x})\right\|-\chi \cdot\left\|\mathbf{h}(\vec{x})-\mathbf{g}_{0}(\vec{x})\right\|-(1-\chi) \cdot\left\|\mathbf{f}(\vec{x})-\mathbf{g}_{0}(\vec{x})\right\| \\
& >m-\chi \cdot \frac{m}{2}-(1-\chi) \cdot \frac{m}{2}=\frac{m}{2} .
\end{aligned}
$$

The magnitude of $\mathbf{g}$ is also bounded above on $\bar{B}^{n}\left(\vec{p}, \frac{\delta}{2}\right)$ :

$$
\begin{aligned}
\|\mathbf{g}(\vec{x})\| & =\|\chi \cdot \mathbf{h}(\vec{x})+(1-\chi) \cdot \mathbf{f}(\vec{x})\| \\
& \leq \chi \cdot\left(\left\|\mathbf{h}(\vec{x})-\mathbf{g}_{0}(\vec{x})\right\|+\left\|\mathbf{g}_{0}(\vec{x})\right\|\right)+(1-\chi) \cdot\|\mathbf{f}(\vec{x})\| \\
& <\chi \cdot\left(\frac{\epsilon}{8}+\frac{\epsilon}{4}\right)+(1-\chi) \cdot \frac{\epsilon}{4}<\frac{\epsilon}{2} .
\end{aligned}
$$

Remark 3.9. The constructions of Corollaries 3.7 and 3.8 are not compatible; it does not immediately follow from them that if $\mathbf{f}$ is both smooth and semialgebraic (for example, polynomial), then there exists $\mathbf{g}$ which is also both smooth and semialgebraic. An analogue of Corollary 3.7 using Weierstrass Approximation could give a polynomial approximation to $\mathbf{f}$, at the cost of losing Property (11) from Theorem 3.6 and shrinking the radius $R$ from (3).

\section{Homotopy THROUGH NONVANISHING MAPS}

The goal of this Section is to perturb a map $\mathbf{f}$ that has a locally inessential zero by a homotopy $F(\vec{x}, t)$ which has no nearby zeros for $t>0$. This is a logically stronger property than the negation of the following stability property: "there exists some $\epsilon>0$ so that for all $t \in[0, \epsilon], F(\vec{x}, t)$ has a zero near $\vec{p}$." In the $q=n$ case, such a homotopy through nonvanishing maps is constructed by $\left[\mathrm{D}_{3}\right.$, where $\mathbf{f}$ and $F$ are continuous (and further, invariant under a group action). The novelty here is the generalization to $q \neq n$, and a construction explicit enough to work in the semialgebraic category, using Corollary 3.8. The main step (14) in the following 
Theorem (and in that of $\mathrm{D}_{3}$ Proposition 1) is analogous to the well-known "Alexander's Trick" in topology (Deloup $)$.

Theorem 4.1. Let $q$, f, $\vec{p}$, and $R$ be as in Theorem 3.6. If $\mathbf{f}$ has a locally inessential zero at $\vec{p}$, then for any $\rho$ with $0<\rho<R$, there exist a continuous map $\mathbf{j}: \Omega \rightarrow \mathbb{R}^{q}$ and a continuous homotopy $F: \Omega \times[0,1] \rightarrow \mathbb{R}^{q}$ from $\mathbf{f}$ to $\mathbf{j}$, such that:

- F fixes the values of $\mathbf{f}$ outside an arbitrarily small ball:

$$
(\vec{x}, t) \in\left(\Omega \backslash B^{n}(\vec{p}, \rho)\right) \times[0,1] \Longrightarrow F(\vec{x}, t)=\mathbf{f}(\vec{x}), \text { and }
$$

- For every non-zero time $t, F(\vec{x}, t)$ is nonvanishing as a function of $\vec{x}$ near $\vec{p}$ :

$$
t>0 \Longrightarrow V(F(\vec{x}, t)) \cap B^{n}(\vec{p}, R)=\varnothing .
$$

If, additionally, $\mathbf{f}$ is continuous and semialgebraic, then there exist $\mathbf{j}$ and $F$ as above which are also continuous and semialgebraic.

Proof. Pick any $\epsilon_{1} \in(0,1]$ and apply the construction of Theorem 3.6 (or Corollary 3.8 in the semialgebraic case) to $\mathbf{f}$ to get a continuous (respectively, semialgebraic) $\mathrm{g}: \Omega \rightarrow \mathbb{R}^{q}$ satisfying (2) and a corresponding $\delta$ with $0<\delta<\min \{1, \rho\}$. By construction, for $\vec{x} \in \bar{B}^{n}\left(\vec{p}, \frac{\delta}{2}\right)$, $\|\mathbf{f}(\vec{x})\|<\frac{\epsilon_{1}}{2}$ and $\|\mathbf{g}(\vec{x})\|<\frac{\epsilon_{1}}{2}$ (using (17) in the semialgebraic case). There is also a lower bound on $\bar{B}^{n}\left(\vec{p}, \frac{\delta}{2}\right),\|\mathbf{g}(\vec{x})\| \geq \frac{m}{2}$, with $m$ from Theorem 3.6, and $\frac{m}{2}$ from Corollary 3.8. After this point, we assume $\vec{p}=\overrightarrow{0} \in \Omega$ just to declutter the formulas.

For each $t \in\left[0, \frac{\delta}{2}\right]$, define continuous, piecewise linear functions $\alpha_{t}:[0, \infty) \rightarrow(0, \infty)$ and $\beta_{t}:[0, \infty) \rightarrow(0, \infty)$, by:

$$
\begin{aligned}
& \alpha_{t}(s)=\left\{\begin{array}{cc}
\frac{\delta}{t^{2}} & 0 \leq s<\frac{t^{2}}{2} \\
\left(\frac{1-\frac{\delta}{t^{2}}}{\frac{t^{2}}{2}}\right) & \left(s-t^{2}\right)+1 \\
1 & \frac{t^{2}}{2} \leq s<t^{2} \\
1 & t^{2} \leq s
\end{array}\right\}, \\
& \beta_{t}(s)=\left\{\begin{array}{cc}
\frac{1-\frac{2 t}{\delta}}{t} s+\frac{2 t}{\delta} & 0 \leq s<t \\
1 & t \leq s
\end{array}\right\} .
\end{aligned}
$$

So $\alpha_{t}$ is weakly decreasing in $s, \beta_{t}$ is weakly increasing, and in particular, for $t=0, \alpha_{0} \equiv \beta_{0} \equiv 1$.

Based on formulas (10) and (11), define the following maps:

$$
\begin{aligned}
\vec{\alpha}(\vec{x}, t) & =\alpha_{t}(\|\vec{x}\|) \cdot \vec{x} \\
\beta(\vec{x}, t) & =\beta_{t}(\|\vec{x}\|) .
\end{aligned}
$$

Both are defined for all $(\vec{x}, t) \in \mathbb{R}^{n} \times \mathbb{R}^{1}$, although neither $\vec{\alpha}$ nor $\beta$ is continuous. Some elementary algebraic expansion of (10) and (11) with $s=\sqrt{x_{1}^{2}+\cdots+x_{n}^{2}}$ will show that the graph of $\vec{\alpha}$ is a semialgebraic set in $\mathbb{R}^{n} \times \mathbb{R}^{1} \times \mathbb{R}^{n}$, and the graph of $\beta$ is a semialgebraic set in $\mathbb{R}^{n} \times \mathbb{R}^{1} \times \mathbb{R}^{1}$.

Define $F_{0}: \Omega \times\left[0, \frac{\delta}{2}\right] \rightarrow \mathbb{R}^{q}$ by:

$$
\begin{aligned}
F_{0}(\vec{x}, t) & =\left\{\begin{array}{l}
\beta_{t}(\|\vec{x}\|) \cdot \mathbf{g}\left(\alpha_{t}(\|\vec{x}\|) \cdot \vec{x}\right) \quad 0 \leq\|\vec{x}\|<\frac{t^{2}}{2} \\
\beta_{t}(\|\vec{x}\|) \cdot \mathbf{f}\left(\alpha_{t}(\|\vec{x}\|) \cdot \vec{x}\right) \quad \frac{t^{2}}{2} \leq\|\vec{x}\|
\end{array}\right\} \\
& =\left\{\begin{array}{ll}
\beta(\vec{x}, t) \cdot \mathbf{g}(\vec{\alpha}(\vec{x}, t)) & 0 \leq\|\vec{x}\|<\frac{t^{2}}{2} \\
\beta(\vec{x}, t) \cdot \mathbf{f}(\vec{\alpha}(\vec{x}, t)) & \frac{t^{2}}{2} \leq\|\vec{x}\|
\end{array}\right\} .
\end{aligned}
$$


By construction, $F_{0}(\vec{x}, 0)=\mathbf{f}(\vec{x})$ for all $x \in \Omega$, and $F_{0}(\vec{x}, t)=\mathbf{f}(\vec{x})$ for all $\vec{x} \in \Omega$ with $\|\vec{x}\|>\frac{\delta}{2}$, so $F_{0}$ satisfies (8). If $\mathbf{f}$ and $\mathbf{g}$ are semialgebraic, then the expression (15) shows that $F_{0}$ is semialgebraic by Proposition 2.6, although it will be easier to work with expression (14).

If $\|\vec{x}\|<\frac{t^{2}}{2}$, then $\left\|\alpha_{t}(\|\vec{x}\|) \cdot \vec{x}\right\|<\frac{\delta}{t^{2}} \cdot \frac{t^{2}}{2}=\frac{\delta}{2}<\delta$, so using the previously mentioned lower and upper bounds for $\|\mathbf{g}(\vec{x})\|$,

$$
0<\frac{2 t}{\delta} \cdot \frac{m}{2}<\left\|F_{0}(\vec{x}, t)\right\|=\beta_{t}(\|\vec{x}\|)\left\|\mathbf{g}\left(\alpha_{t}(\|\vec{x}\|) \cdot \vec{x}\right)\right\|<\frac{\epsilon_{1}}{2} .
$$

If $\frac{t^{2}}{2} \leq\|\vec{x}\|<t^{2}$, then $\frac{t^{2}}{2} \leq\|\vec{x}\| \leq\left\|\alpha_{t}(\|\vec{x}\|) \cdot \vec{x}\right\|<\frac{\delta}{t^{2}} \cdot t^{2}=\delta$. If $t^{2} \leq\|\vec{x}\|<\delta$, then $\left\|\alpha_{t}(\|\vec{x}\|) \cdot \vec{x}\right\|=\|\vec{x}\|<\delta$, so

$$
\begin{aligned}
0 & \leq \frac{2 t}{\delta} \min \left\{\|\mathbf{f}(\vec{x})\|: \frac{t^{2}}{2} \leq\|\vec{x}\|<\delta\right\} \\
& \leq\left\|F_{0}(\vec{x}, t)\right\|=\beta_{t}(\|\vec{x}\|)\left\|\mathbf{f}\left(\alpha_{t}(\|\vec{x}\|) \cdot \vec{x}\right)\right\|<\frac{\epsilon_{1}}{2},
\end{aligned}
$$

with equality in (16) only at $t=0$.

So, the nonvanishing property (9) holds for $F_{0}$, together with an approximation property analogous to (2):

$$
\left\|\mathbf{f}(\vec{x})-F_{0}(\vec{x}, t)\right\| \leq\|\mathbf{f}(\vec{x})\|+\left\|F_{0}(\vec{x}, t)\right\|<\frac{\epsilon_{1}}{2}+\frac{\epsilon_{1}}{2} .
$$

The two pieces of the formula (14) agree on their common boundary where $\|\vec{x}\|=\frac{t^{2}}{2}>0$, $\alpha_{t}(\|\vec{x}\|)=\frac{\delta}{t^{2}}$, and $\left\|\alpha_{t}(\|\vec{x}\|) \cdot \vec{x}\right\|=\frac{\delta}{2}$, so $\alpha_{t}(\|\vec{x}\|) \cdot \vec{x} \in S^{n-1}\left(\vec{p}, \frac{\delta}{2}\right)$. It follows from the continuity of (10) and (11) in $s>0$ and $t>0$ that (12), (13), and $F_{0}$ are continuous for $\vec{x} \neq \overrightarrow{0}$ and $t>0$. For a point $(\vec{x}, t)=\left(\overrightarrow{0}, t_{0}\right)$ with $t_{0}>0$, there is a neighborhood where

$$
F_{0}(\vec{x}, t)=\left(\frac{1-\frac{2 t}{\delta}}{t}\|\vec{x}\|+\frac{2 t}{\delta}\right) \cdot \mathbf{g}\left(\frac{\delta}{t^{2}} \cdot \vec{x}\right),
$$

which is continuous. For a point $(\vec{x}, t)=\left(\vec{x}_{0}, 0\right)$ with $\vec{x}_{0} \neq \overrightarrow{0}$, there is a neighborhood where

$$
F_{0}(\vec{x}, t)=1 \cdot \mathbf{f}(1 \cdot \vec{x})
$$

which is also continuous. It remains only to check continuity at $(\vec{x}, t)=(\overrightarrow{0}, 0)$, where $F_{0}(\overrightarrow{0}, 0)=$ 0 .

For any $\epsilon_{2}>0$, there is some $\delta_{1}>0$ so that if $\|\vec{x}\|<\delta_{1}$, then $\|\mathbf{f}(\vec{x})\|<\epsilon_{2}$. Let $\delta_{2}=\min \left\{\delta, \delta_{1}\right\}$, and let $\delta_{3}=\frac{\epsilon_{2}}{1+\frac{2}{\delta}}>0$. Continuity of $F_{0}$ at the origin will follow from showing that if $\|\vec{x}\|<\delta_{2}$ and $t<\delta_{3}$, then $\left\|F_{0}(\vec{x}, t)\right\|<\epsilon_{2}$. There are three cases.

If $\|\vec{x}\|<\min \left\{\frac{t^{2}}{2}, \delta_{2}\right\}$, then

$$
\begin{aligned}
\left\|F_{0}(\vec{x}, t)\right\| & =\left(\frac{1-\frac{2 t}{\delta}}{t}\|\vec{x}\|+\frac{2 t}{\delta}\right)\left\|\mathbf{g}\left(\frac{\delta}{t^{2}} \cdot \vec{x}\right)\right\| \\
& <\left(\frac{\left(1-\frac{2 t}{\delta}\right) t}{2}+\frac{2 t}{\delta}\right) \frac{\epsilon_{1}}{2}<\left(\frac{\left(1-\frac{2 t}{\delta}\right)}{2}+\frac{2}{\delta}\right) \delta_{3} \frac{1}{2}<\epsilon_{2} .
\end{aligned}
$$

If $\frac{t^{2}}{2} \leq\|\vec{x}\|<\min \left\{t^{2}, \delta_{2}\right\}$, then

$$
\begin{aligned}
\left\|F_{0}(\vec{x}, t)\right\| & =\left(\frac{1-\frac{2 t}{\delta}}{t}\|\vec{x}\|+\frac{2 t}{\delta}\right)\left\|\mathbf{f}\left(\alpha_{t}(\|\vec{x}\|) \cdot \vec{x}\right)\right\| \\
& <\left(\left(1-\frac{2 t}{\delta}\right) t+\frac{2 t}{\delta}\right) \frac{\epsilon_{1}}{2}<\left(\left(1-\frac{2 t}{\delta}\right)+\frac{2}{\delta}\right) \delta_{3} \frac{1}{2}<\epsilon_{2} .
\end{aligned}
$$


If $t^{2} \leq\|\vec{x}\|<\delta_{2}$ then

$$
\left\|F_{0}(\vec{x}, t)\right\|=\beta_{t}(\|\vec{x}\|)\|\mathbf{f}(1 \cdot \vec{x})\|<1 \epsilon_{2}
$$

So $F_{0}$ is a continuous homotopy from $\mathbf{f}(\vec{x})$ to $\mathbf{j}(\vec{x})=F_{0}\left(\vec{x}, \frac{\delta}{2}\right)$ for $0<t \leq \frac{\delta}{2}$; re-scaling the $t$ variable to the interval $[0,1]$ gives $F$ and $\mathbf{j}$ satisfying (8) and (9) as claimed.

Remark 4.2. It would be easy to construct another homotopy from $\mathbf{j}$ to a smooth map $\mathbf{j}_{2}$, by a continuous homotopy of nonvanishing approximations. Concatenation would then give a continuous homotopy $F$ from the original map $\mathbf{f}$ to the smooth map $\mathbf{j}_{2}$. If $\mathbf{f}$ were also smooth, then the existence of a continuous homotopy implies the existence of a smooth homotopy $\tilde{F}$ from $\mathbf{f}$ to $\mathbf{j}_{2}$ ([L] Theorem 6.29), and Property (8) could be arranged. However, finding a smooth, or even $\mathcal{C}^{1}$, homotopy also satisfying (9) seems to be a difficult problem, which we state here as a Question.

Question 4.3. Let $q, \mathbf{f}, \vec{p}$, and $R$ be as in Theorem 3.6 , where $\mathbf{f}$ has a locally inessential zero at $\vec{p}$. Suppose $\mathbf{f}$ is $\left[\mathcal{C}^{1}\right.$, smooth, real analytic, polynomial] on $\Omega$. Does there always exist a $\left[\mathcal{C}^{1}\right.$, smooth, real analytic, polynomial] map $F: B^{n}(\vec{p}, \rho) \times(-1,1) \rightarrow \mathbb{R}^{q}$ for some $0<\rho<R$ with the following properties?

- $F$ agrees with $\mathbf{f}$ for all $\vec{x} \in B^{n}(\vec{p}, \rho)$ at time $t=0: F(\vec{x}, 0)=\mathbf{f}(\vec{x})$, and

- for every non-zero time $t, F(\vec{x}, t)$ is nonvanishing as a function of $\vec{x}$ : $t \neq 0 \Longrightarrow$ $V(F(\vec{x}, t))=\varnothing$.

The Question can be considered as a classic extension problem: does a $\mathbb{R}^{q}$ valued $\mathbf{f}$ with a locally inessential zero in $\mathbb{R}^{n}$ extend, locally, to a $\mathbb{R}^{q}$ valued function $F$ with an isolated zero in $\mathbb{R}^{n+1}$, with the same regularity? Theorem 4.1 constructs such a continuous one-sided extension for $0 \leq t \leq 1$; then $F\left(\vec{x}, t^{2}\right)$ for $-1 \leq t \leq 1$ is a two-sided extension. This also solves the two-sided extension problem in the continuous, semialgebraic case.

We are not conjecturing an answer either way to any of the stated versions of Question 4.3. it would be interesting to get either a proof of "yes" or a concrete counterexample for "no" for any of the cases. It is possible that the answer will depend on the dimensions $n$ and $q$, in analogy with exotic smoothness phenomena.

Corollary 4.4. Given $n$ and $q$, if the answer to Question 4.3 is "yes" in the case where "real analytic" is stated in both the hypothesis and the conclusion, then the answer is also yes in the polynomial case.

Proof. Assume as before that $\vec{p}=\overrightarrow{0}$. Suppose that $\mathbf{f}(\vec{x})$ as in Question 4.3 is a polynomial map with an isolated zero at $\overrightarrow{0}$, which extends to a real analytic $F(\vec{x}, t)$ on some $B^{n}(\overrightarrow{0}, \rho) \times(-1,1)$ with an isolated zero at $(\overrightarrow{0}, 0)$. By the Łojasiewicz inequality, there are some positive constants $C_{1}$ and $\eta$ so that in some possibly smaller neighborhood of $(\overrightarrow{0}, 0)$,

$$
\|F(\vec{x}, t)\| \geq C_{1}\|(\vec{x}, t)\|^{\eta} .
$$

Choose an integer $D \geq \max \{\eta$, degree $(\mathbf{f})\}$; then $F$ has a degree $D$ Taylor polynomial $P(\vec{x}, t)$ that satisfies $P(\vec{x}, 0)=\mathbf{f}(\vec{x})$, and there is some constant $C_{2}>0$ so that in some neighborhood of the origin, $\|F(\vec{x}, t)-P(\vec{x}, t)\| \leq C_{2}\|(\vec{x}, t)\|^{D+1}$. So, $P$ also has a unique zero in a sufficiently small neighborhood of $(\overrightarrow{0}, 0)$ :

$$
\begin{aligned}
\|P(\vec{x}, t)\| & \geq\|F(\vec{x}, t)\|-\|F(\vec{x}, t)-P(\vec{x}, t)\| \\
& \geq C_{1}\|(\vec{x}, t)\|^{\eta}-C_{2}\|(\vec{x}, t)\|^{D+1} \\
& =C_{1}\|(\vec{x}, t)\|^{\eta}\left(1-\frac{C_{2}}{C_{1}}\|(\vec{x}, t)\|^{D+1-\eta}\right) .
\end{aligned}
$$


The $t$ variable can be re-scaled as needed.

The converse of Theorem 4.1 also holds (in the continuous case), so this gives a sixth equivalent property for Lemma 3.1.

Theorem 4.5. For a continuous map $\mathbf{f}: \Omega \rightarrow \mathbb{R}^{q}$, and an isolated zero $\vec{p} \in B^{n}(\vec{p}, R)$ as in Definition 2.3, the following are equivalent:

- $\vec{p}$ is a locally inessential zero of $\mathbf{f}$;

- There exist some $r_{0} \in(0, R)$ and a continuous $F: B^{n}\left(\vec{p}, r_{0}\right) \times[0,1] \rightarrow \mathbb{R}^{q}$ such that $F(\vec{x}, 0)=\mathbf{f}(\vec{x})$ for $\vec{x} \in B^{n}\left(\vec{p}, r_{0}\right)$ and $t>0 \Longrightarrow F(\vec{x}, t) \neq \mathbf{0}$.

Proof. The existence of such a homotopy $F$ implies Property (iv) from Lemma 3.1, as follows. For any $\epsilon>0$, there is some $\delta$ with $0<\delta \leq \min \left\{1, r_{0}\right\}$ and so that if $\|\vec{x}-\vec{p}\|<\delta$ and $0 \leq t<\delta$, then $\|F(\vec{x}, t)\|<\epsilon$. For any $r \in(0, \delta)$, define $\Theta_{r}: B_{*}^{n}(\vec{p}, r) \times[0,1] \rightarrow \mathbb{R}^{q} \backslash\{\mathbf{0}\}$ by $\Theta_{r}(\vec{x}, t)=F\left(\vec{p}+(1-t) \cdot(\vec{x}-\vec{p}), \frac{\delta}{2} t\right)$. Then, $\Theta_{r}(\vec{x}, 0)=F(\vec{x}, 0)=\mathbf{f}(\vec{x}), \Theta_{r}(\vec{x}, 1) \equiv F\left(\vec{p}, \frac{\delta}{2}\right)$, and $\Theta_{r}(\vec{x}, t) \in B_{*}^{q}(\mathbf{0}, \epsilon)$ by construction.

\section{REAL ANALYTiC MAPS TO THE PLANE}

The following Theorem gives an answer to a version of Question 4.3 in the special case where the target dimension is $q=2$ and the given data $\mathbf{f}$ is real analytic on $\Omega \subseteq \mathbb{R}^{n}$, for any $n \geq 1$. The construction of a Hölder continuous extension $F$ which is real analytic except at the origin, where it satisfies a one-point Lipschitz condition $\|F(\vec{x}, t)\| \leq C\|(\vec{x}-\vec{p}, t)\|$, does not use the Theorems of Sections 3 or 4 . One step refers to Lemma 5.3, which appears after the main Proof.

Theorem 5.1. Let $\mathbf{f}: \Omega \rightarrow \mathbb{R}^{2}$ be a real analytic map so that $\mathbf{f}$ has an isolated zero $\vec{p} \in \Omega$. If either:

- $n \neq 2$; or,

- $n=2$ and $\vec{p}$ is a locally inessential zero of $\mathbf{f}$,

then there exist some $\rho>0$, and a Hölder continuous map $F: B^{n+1}((\vec{p}, 0), \rho) \rightarrow \mathbb{R}^{2}$ such that:

- F locally extends $\mathbf{f}: F(\vec{x}, 0)=\mathbf{f}(\vec{x})$ for $\vec{x} \in B^{n}(\vec{p}, \rho)$, and

- on the punctured ball $B_{*}^{n+1}((\vec{p}, 0), \rho), F$ is real analytic and nonvanishing, and

- there is some $C>0$ so that for all $(\vec{x}, t) \in B_{*}^{n+1}((\vec{p}, 0), \rho)$,

$$
\|F(\vec{x}, t)\| \leq C\|(\vec{x}-\vec{p}, t)\| .
$$

Proof. Let $\vec{p}=\overrightarrow{0}$ and let $R>0$, as in Theorem 3.6. Consider $\mathbf{f}$ as a complex valued function $\mathbf{f}(\vec{x})=\left(f_{1}(\vec{x}), f_{2}(\vec{x})\right)=f_{1}+i f_{2}$.

Case 1. We first consider the case $n=1$, where there is a simple proof and a stronger result, neither of which we have been able to generalize to higher $n$ or $q$. By the real analytic assumption, there is some $\rho_{0}>0$ so that for $\left|x_{1}\right|<\rho_{0}, g\left(x_{1}\right)=f_{1}\left(x_{1}\right)+i f_{2}\left(x_{1}\right)$ is equal to a non-constant convergent series with complex coefficients $\sum_{k=1}^{\infty}\left(a_{k}+i b_{k}\right) x_{1}^{k}$. Replacing the real variable $x_{1}$ with a complex variable $z=x_{1}+i t$ gives, for the same radius $|z|<\rho_{0}$, a series converging to a holomorphic function $F(z)$ on $B^{2}\left((0,0), \rho_{0}\right) \subseteq \mathbb{C}$ which extends $g=f_{1}+i f_{2}$ and whose zeros are all isolated.

Case 2. $n \geq 2$. The first three steps in the proof are preparation steps for $\mathbf{f}$ in a small neighborhood; Step 4 constructs a $k^{\text {th }}$ root, and the extension is constructed in the remaining 
steps. The locally inessential property is used only in Step 1, although as mentioned in Remark 3.5. any isolated zero is locally inessential in the $n \neq 2$ case, by Lemma 3.4.

Step 1. (Normalization on a boundary sphere) Using Property (iv) from Lemma 3.1, there exists some $0<\rho_{1}<\min \left\{R, \frac{1}{2}\right\}$ so that $\mathbf{f}$ restricted to $B_{*}^{n}\left(\overrightarrow{0}, \rho_{1}\right) \rightarrow \mathbb{C} \backslash\{0+0 i\}$ is homotopic in $\mathbb{C} \backslash\{0+0 i\}$ to the constant map with image $\{1+0 i\}$. By the usual construction of the universal covering space exp $: \mathbb{C} \rightarrow \mathbb{C} \backslash\{0+0 i\}$, there is a branch of the logarithm and a lift of $\mathbf{f}\left([\underline{S}]\right.$ Theorem 2.4.5) to a real analytic composite $\log$ of : $B_{*}^{n}\left(\overrightarrow{0}, \rho_{1}\right) \rightarrow \mathbb{C}$ so that $\exp (\log (\mathbf{f}(\vec{x})))=\mathbf{f}(\vec{x})$. For any $0<\rho_{2}<\rho_{1}$, the restriction of $\log$ of to the sphere $S^{n-1}\left(\overrightarrow{0}, \rho_{2}\right)$ is real analytic, and solving the classical Dirichlet problem gives a function $\mathbf{u}(\vec{x})$ which is harmonic and real analytic on some ball $B^{n}\left(\overrightarrow{0}, \rho_{3}\right), \rho_{2}<\rho_{3}<\rho_{1}$, and such that $\mathbf{u}(\vec{x})=\log (\mathbf{f}(\vec{x}))$ for $\vec{x} \in S^{n-1}\left(\overrightarrow{0}, \rho_{2}\right)$. Let $\mathbf{m}(\vec{x})=\exp (-\mathbf{u}(\vec{x}))$, so by construction, the complex product $\mathbf{m} \cdot \mathbf{f}$ is real analytic on $B^{n}\left(\overrightarrow{0}, \rho_{3}\right), \mathbf{m}(\vec{x}) \mathbf{f}(\vec{x}) \equiv 1+0 i$ for all $\vec{x} \in S^{n-1}\left(\overrightarrow{0}, \rho_{2}\right),(\mathbf{m} \cdot \mathbf{f})^{-1}(\{0+0 i\})=\{\overrightarrow{0}\}$, and for $n=2, \mathbf{m} \cdot \mathbf{f}$ still has a locally inessential zero.

Step 2. (Polar coordinates) Using the exponential covering space again with base point

$$
(\mathbf{m} \cdot \mathbf{f})\left(\rho_{2}, 0, \ldots, 0\right)=1+0 i
$$

there is a branch of the logarithm on $B_{*}^{n}\left(\overrightarrow{0}, \rho_{3}\right)$ with real and imaginary parts:

$$
\log ((\mathbf{m} \cdot \mathbf{f})(\vec{x}))=\ln |(\mathbf{m} \cdot \mathbf{f})(\vec{x})|+i \theta(\vec{x}),
$$

where $\theta\left(\rho_{2}, 0, \ldots, 0\right)=0$. Exponentiating,

$$
(\mathbf{m} \cdot \mathbf{f})(\vec{x})=r(\vec{x}) \cdot \exp (i \theta(\vec{x})),
$$

for $\theta$ and $r>0$ real analytic on $B_{*}^{n}\left(\overrightarrow{0}, \rho_{3}\right)$.

Step 3. (Boundedness of $\theta$ ) Using (19), the real analytic function $\operatorname{Re}(\mathbf{m} \cdot \mathbf{f})$ is not identically zero on any open interval of the $x_{1}$-axis $\left\{\left(x_{1}, 0, \ldots, 0\right)\right\} \cap B^{n}\left(\overrightarrow{0}, \rho_{3}\right)$. So, by the Weierstrass Preparation Theorem, there exist some radius $0<\rho_{4}<\rho_{2}$, some degree $N$, and some real analytic functions $\nu, c_{1}, \ldots, c_{N}$, so that for all $\vec{x} \in B^{n}\left(\overrightarrow{0}, \rho_{4}\right)$,

$$
\operatorname{Re}(\mathbf{m}(\vec{x}) \mathbf{f}(\vec{x}))=\nu(\vec{x}) \cdot\left(x_{1}^{N}+c_{1}\left(x_{2}, \ldots, x_{n}\right) x_{1}^{N-1}+\ldots+c_{N}\left(x_{2}, \ldots, x_{n}\right)\right),
$$

where $\nu(\vec{x})$ is nonvanishing. Denote the open cylinder

$$
\Gamma=\left(-\frac{\rho_{4}}{2}, \frac{\rho_{4}}{2}\right) \times B^{n-1}\left(\overrightarrow{0}, \frac{\rho_{4}}{2}\right) \subseteq B^{n}\left(\overrightarrow{0}, \rho_{4}\right) .
$$

On the sphere $S^{n-1}\left(\overrightarrow{0}, \rho_{2}\right), \theta \equiv 0$, and on the complement $\bar{B}^{n}\left(\overrightarrow{0}, \rho_{2}\right) \backslash \Gamma, \theta$ is bounded by compactness: $|\theta| \leq K$. For a fixed $x^{\prime}=\left(x_{2}^{\prime}, \ldots, x_{n}^{\prime}\right) \in B^{n-1}\left(\overrightarrow{0}, \frac{\rho_{4}}{2}\right)$, consider the following expression as a function of $x_{1}$ only:

$$
\begin{aligned}
\operatorname{Re}\left(\mathbf{m}\left(x_{1}, x^{\prime}\right) \mathbf{f}\left(x_{1}, x^{\prime}\right)\right) & =\nu\left(x_{1}, x^{\prime}\right)\left(x_{1}^{N}+c_{1}\left(x^{\prime}\right) x_{1}^{N-1}+\ldots+c_{N}\left(x^{\prime}\right)\right) \\
& =\left|\mathbf{m}\left(x_{1}, x^{\prime}\right) \mathbf{f}\left(x_{1}, x^{\prime}\right)\right| \cos \left(\theta\left(x_{1}, x^{\prime}\right)\right) \quad\left(\text { for }\left(x_{1}, x^{\prime}\right) \neq \overrightarrow{0}\right) .
\end{aligned}
$$

By the Fundamental Theorem of Algebra, for a fixed $x^{\prime} \in B_{*}^{n-1}\left((0, \ldots, 0), \frac{\rho_{4}}{2}\right), \cos \left(\theta\left(x_{1}, x^{\prime}\right)\right)$ can have at most $N$ zeros on the interval $-\frac{\rho_{4}}{2}<x_{1}<\frac{\rho_{4}}{2}$, so $\left|\theta\left(x_{1}, x^{\prime}\right)\right|$ is bounded by $K+2 \pi N$ for all $x_{1}$ with $\left(x_{1}, x^{\prime}\right) \in \bar{B}^{n}\left(\overrightarrow{0}, \rho_{2}\right)$, and this bound does not depend on $x^{\prime}$. For points on the $x_{1}$ axis, where $x_{1} \neq 0$ and $x^{\prime}=(0, \ldots, 0),|\theta|$ has the same bound by continuity. By continuity at points on the boundary, there is some radius $\rho_{5}, \rho_{2}<\rho_{5}<\rho_{3}$, so that $\theta$ is bounded on $\bar{B}_{*}^{n}\left(\overrightarrow{0}, \rho_{5}\right)$. 
Step 4. (Taking a root) By the boundedness of $\theta$, there is some integer $k$ so that $\frac{|\theta(\vec{x})|}{k}<\frac{\pi}{2}$ for $\vec{x} \in \vec{B}_{*}^{n}\left(\overrightarrow{0}, \rho_{5}\right)$. The following $k^{t h}$ root is well-defined and continuous on $\bar{B}^{n}\left(\overrightarrow{0}, \rho_{5}\right)$ :

$$
(\mathbf{m}(\vec{x}) \mathbf{f}(\vec{x}))^{1 / k}=\left\{\begin{array}{cc}
(r(\vec{x}))^{1 / k} \exp (i \theta(\vec{x}) / k) & \vec{x} \neq \overrightarrow{0} \\
0 & \vec{x}=\overrightarrow{0}
\end{array}\right\}
$$

and satisfies

$$
\operatorname{Re}\left((\mathbf{m}(\vec{x}) \mathbf{f}(\vec{x}))^{1 / k}\right) \geq 0 .
$$

In the open set $B_{*}^{n}\left(\overrightarrow{0}, \rho_{5}\right),(\mathbf{m}(\vec{x}) \mathbf{f}(\vec{x}))^{1 / k}$ is real analytic and nonvanishing (with positive real part), and is $\equiv 1+0 i$ on $S^{n-1}\left(\overrightarrow{0}, \rho_{2}\right)$.

Because $\mathbf{m} \cdot \mathbf{f}$ is real analytic on $B^{n}\left(\overrightarrow{0}, \rho_{3}\right)$, its components have bounded gradient on the closed ball $\bar{B}^{n}\left(\overrightarrow{0}, \rho_{5}\right)$ and $\mathbf{m} \cdot \mathbf{f}$ satisfies a uniform Lipschitz condition on $B^{n}\left(\overrightarrow{0}, \rho_{5}\right)$. Lemma 5.3 applies, to show that $(\mathbf{m}(\vec{x}) \mathbf{f}(\vec{x}))^{1 / k}$ is Hölder continuous on $B^{n}\left(\overrightarrow{0}, \rho_{5}\right)$ with exponent $\frac{1}{k}$.

Step 5. (Solving a boundary value problem) Now consider $\mathbb{R}^{n+1}$ with coordinates $(\vec{x}, t)$, and the closed ball $\bar{B}^{n}\left(\overrightarrow{0}, \rho_{2}\right) \times\{0\}$ as the equatorial disk of the closed ball $\bar{B}^{n+1}\left((\overrightarrow{0}, 0), \rho_{2}\right)$. Let $S_{+}^{n}\left((\overrightarrow{0}, 0), \rho_{2}\right)$ denote the upper hemisphere as in Notation 2.1 , so that the boundary of the upper half ball $B_{+}^{n+1}\left((\overrightarrow{0}, 0), \rho_{2}\right)$ is the union $S_{+}^{n}\left((\overrightarrow{0}, 0), \rho_{2}\right) \cup\left(\vec{B}^{n}\left(\overrightarrow{0}, \rho_{2}\right) \times\{0\}\right)$. On this boundary set, the following function is continuous:

$$
\mathbf{h}(\vec{x}, t)=\left\{\begin{array}{cl}
(\mathbf{m}(\vec{x}) \mathbf{f}(\vec{x}))^{1 / k} & \vec{x} \in \bar{B}^{n}\left(\overrightarrow{0}, \rho_{2}\right), t=0 \\
1+0 i & (\vec{x}, t) \in S_{+}^{n}\left((\overrightarrow{0}, 0), \rho_{2}\right)
\end{array}\right\} .
$$

Solving the classical Dirichlet problem extends $\mathbf{h}$ to a complex valued continuous function $H(\vec{x}, t)$ on the closure of $B_{+}^{n+1}\left((\overrightarrow{0}, 0), \rho_{2}\right)$ such that $H(\vec{x}, t)$ is harmonic on $B_{+}^{n+1}\left((\overrightarrow{0}, 0), \rho_{2}\right)$. By the maximum principle applied to the harmonic real function $-\operatorname{Re}(H(\vec{x}, t))$ and (20), $\operatorname{Re}(H(\vec{x}, t))$ is strictly positive on the interior and attains its minimum value 0 only at the origin. Near boundary points $(\vec{x}, 0) \in B_{*}^{n}\left(\overrightarrow{0}, \rho_{2}\right) \times\{0\}, H$ extends uniquely and real analytically across the boundary into the lower half space $\{t<0\}$. For any $\rho_{6}$ with $0<\rho_{6}<\rho_{2}$, the restriction of $H(\vec{x}, t)$ to the closure of the smaller half ball $B_{+}^{n+1}\left((\overrightarrow{0}, 0), \rho_{6}\right)$ is Hölder continuous with the same exponent, $\alpha=\frac{1}{k}$, as the data on the flat part of the boundary.

Step 6. (Constructing the extension) The composite $H_{2}(\vec{x}, t)=H\left(\vec{x}, t^{2}\right)$ is defined and Hölder continuous on the whole ball $B^{n+1}\left((\overrightarrow{0}, 0), \rho_{6}\right)$ : using $\rho_{6}<\frac{1}{2}$,

$$
\begin{aligned}
\left|H_{2}(\vec{y}, s)-H_{2}(\vec{x}, t)\right| & =\left|H\left(\vec{y}, s^{2}\right)-H\left(\vec{x}, t^{2}\right)\right| \\
& \leq C_{3}\left\|\left(\vec{y}, s^{2}\right)-\left(\vec{x}, t^{2}\right)\right\|^{1 / k} \leq C_{3}\left[\|\vec{x}-\vec{y}\|^{2}+\left|s^{2}-t^{2}\right|^{2}\right]^{1 /(2 k)} \\
& \leq C_{3}\left[\|\vec{x}-\vec{y}\|^{2}+|s-t|^{2}\right]^{1 /(2 k)} \leq C_{3}\|(\vec{y}, s)-(\vec{x}, t)\|^{1 / k} .
\end{aligned}
$$

$H\left(\vec{x}, t^{2}\right)$ is real analytic except at the origin, at which the Hölder condition gives, for all $(\vec{x}, t)$ in $B^{n+1}\left((\overrightarrow{0}, 0), \rho_{6}\right)$ :

$$
\left|H_{2}(\vec{x}, t)\right|=\left|H\left(\vec{x}, t^{2}\right)\right| \leq C_{3}\left\|\left(\vec{x}, t^{2}\right)\right\|^{1 / k} \leq C_{3}\|(\vec{x}, t)\|^{1 / k} .
$$

The $k^{\text {th }}$ power $\left(H\left(\vec{x}, t^{2}\right)\right)^{k}$ is similarly Hölder continuous with the same exponent $\frac{1}{k}$ on the same ball, and real analytic except at the origin, and from (21), it satisfies:

$$
\left|\left(H\left(\vec{x}, t^{2}\right)\right)^{k}\right| \leq C_{3}^{k}\|(\vec{x}, t)\| .
$$


By construction, $\left(H\left(\vec{x}, t^{2}\right)\right)^{k}$ has a unique zero at $(\overrightarrow{0}, 0)$, and for $t=0,(H(\vec{x}, 0))^{k}=\mathbf{m}(\vec{x}) \mathbf{f}(\vec{x})$. An extension $F$ of $\mathbf{f}$ as claimed, with $\rho=\rho_{6}$ and satisfying (18), is:

$$
F(\vec{x}, t)=\frac{\left(H\left(\vec{x}, t^{2}\right)\right)^{k}}{\mathbf{m}(\vec{x})} .
$$

Such an extension $F$ may vanish to higher order but would still not necessarily have a continuous derivative at the origin; the above argument also does not show that $F$ is (uniformly) Lipschitz continuous in a neighborhood of the origin.

Remark 5.2. The Proof of Theorem 5.1 used some facts about harmonic functions that are well-known to PDE experts. The existence of a solution of the Dirichlet problem, to construct $\mathbf{u}$ in Step 1, and to construct $H$ in Step 5, is given by Theorem 2.14 of GT, using only that the boundary data is continuous and the domain has a sufficiently regular boundary. The maximum principle (which was the key step for the nonvanishing) holds for any bounded domain. The fact that $\mathbf{u}$ and $H$ extend real analytically across the boundary in neighborhoods where the boundary and the Dirichlet data are real analytic follows from a standard argument using the Cauchy-Kovalevskaya Theorem and the reflection principle for harmonic functions ( $\mathrm{G}]$ ). The $\mathcal{C}^{0, \alpha}$ Hölder property for the harmonic function $H(\vec{x}, t)$ up to a part of the boundary where the boundary values are Hölder continuous, from Step 5, is the deepest result used in the Proof; it also depends, in general, on the geometry of the boundary ([Aikawa, [LU], [M]).

Lemma 5.3. Given $R>0$, integers $n \geq 1, k \geq 1$, and a continuous function $\mathbf{g}: B^{n}(\overrightarrow{0}, R) \rightarrow \mathbb{C}$ such that $\operatorname{Re}(\mathbf{g}(\vec{x})) \geq 0$, with equality only at $\mathbf{g}(\overrightarrow{0})=0+0$, if the $k^{\text {th }}$ power $(\mathbf{g}(\vec{x}))^{k}$ is Lipschitz continuous on $B^{n}(\overrightarrow{0}, R)$ : for some $C_{1}$ and any $\vec{x}, \vec{y} \in B^{n}(\overrightarrow{0}, R)$,

$$
\left|(\mathbf{g}(\vec{y}))^{k}-(\mathbf{g}(\vec{x}))^{k}\right| \leq C_{1}\|\vec{y}-\vec{x}\|
$$

then $\mathbf{g}$ is Hölder continuous on $B^{n}(\overrightarrow{0}, R)$ : for some $C_{2}$ and any $\vec{x}, \vec{y} \in B^{n}(\overrightarrow{0}, R)$,

$$
|\mathbf{g}(\vec{y})-\mathbf{g}(\vec{x})| \leq C_{2}\|\vec{y}-\vec{x}\|^{1 / k} .
$$

Proof. For $z \in \mathbb{C} \backslash\{0+0 i\}$, denote by $\operatorname{Arg}(z)$ the angle $\vartheta \in(-\pi, \pi]$ so that $z=|z| e^{i \vartheta}$.

Step 1. Claim: For $z, w \in \mathbb{C} \backslash\{0+0 i\}$, if $\left|\operatorname{Arg}\left(\frac{z}{w}\right)\right|<\frac{\pi}{2 k}$ then $|w-z|^{k} \leq\left|w^{k}-z^{k}\right|$, with equality only if $k=1$ or $z=w$. The Claim is trivial for $k=1$; for $k>1$, the proof of the Claim has two cases.

Case 1. $|z| \leq|w|$. Let $\zeta=\frac{z}{w}$. By the hypothesis $|\operatorname{Arg}(\zeta)|<\frac{\pi}{2 k}$, for $j=0,1, \ldots, k-1$, $\operatorname{Re}\left(\zeta^{j}\right)>0$, so $\operatorname{Re}\left(1+\zeta+\cdots+\zeta^{k-1}\right)>1$ and $\left|1+\zeta+\cdots+\zeta^{k-1}\right|>1 .|\operatorname{Arg}(\zeta)|<\frac{\pi}{2 k}$ and $|\zeta|<1$ also imply $|1-\zeta|<1$, so

$$
|1-\zeta|^{k} \leq|1-\zeta| \leq|1-\zeta|\left|1+\zeta+\cdots+\zeta^{k-1}\right|=\left|1-\zeta^{k}\right|
$$

with equality only if $\zeta=1$. Multiplying both sides by $|w|^{k}$ establishes the Claim.

Case 2. $|w|<|z|$. Let $\zeta=\frac{w}{z}$, so $|\operatorname{Arg}(\zeta)|<\frac{\pi}{2 k}$ and $|\zeta|<1$ still hold, so (23) follows, and then multiplying by $|z|^{k}$ establishes the Claim.

Step 2. The property (22) clearly holds for $\vec{x}=\vec{y}$ or $k=1$, so the following cases will assume $k>1$ and $\vec{x} \neq \vec{y}$.

Case 1. For $\vec{x}=\overrightarrow{0}$, where $\mathbf{g}(\vec{x})=0+0 i$, the conclusion $|\mathrm{g}(\vec{y})| \leq C_{2}\|\vec{y}\|^{1 / k}$ follows from the assumption $\left|(\mathbf{g}(\vec{y}))^{k}-(0+0 i)^{k}\right| \leq C_{1}\|\vec{y}-\overrightarrow{0}\|$, with $C_{2}=C_{1}^{1 / k}$. The case $\vec{y}=\overrightarrow{0}$ is analogous. 
Case 2. If $\vec{y}=\lambda \vec{x}$ for some $\lambda<0$, so that $\overrightarrow{0}$ is between $\vec{x}$ and $\vec{y}$ in $B^{n}(\overrightarrow{0}, R)$, then $\|\vec{x}\|<\|\vec{y}-\vec{x}\|$ and $\|\vec{y}\|<\|\vec{y}-\vec{x}\|$. Then using Case 1 .,

$$
|\mathbf{g}(\vec{y})-\mathbf{g}(\vec{x})| \leq|\mathbf{g}(\vec{y})|+|\mathbf{g}(\vec{x})| \leq C_{1}^{1 / k}\|\vec{y}\|^{1 / k}+C_{1}^{1 / k}\|\vec{x}\|^{1 / k}<2 C_{1}^{1 / k}\|\vec{y}-\vec{x}\|^{1 / k} .
$$

For the remaining cases, with $\vec{x}, \vec{y} \in B_{*}^{n}(\overrightarrow{0}, R)$, let $z=\mathbf{g}(\vec{x})$ and $w=\mathbf{g}(\vec{y})$, so $\operatorname{Re}(z)>0$ and $\operatorname{Re}(w)>0$ by hypothesis, and $\operatorname{Arg}\left(\frac{z}{w}\right) \in(-\pi, \pi)$.

Case 3. If $\left|\operatorname{Arg}\left(\frac{z}{w}\right)\right|<\frac{\pi}{2 k}$, then the Claim from Step 1. applies and

$$
|\mathbf{g}(\vec{y})-\mathbf{g}(\vec{x})|^{k} \leq\left|(\mathbf{g}(\vec{y}))^{k}-(\mathbf{g}(\vec{x}))^{k}\right| \leq C_{1}\|\vec{y}-\vec{x}\|
$$

which gives (22) with $C_{2}=C_{1}^{1 / k}$.

Case 4. Suppose $\left|\operatorname{Arg}\left(\frac{z}{w}\right)\right| \geq \frac{\pi}{2 k}$ and the line segment connecting $\vec{x}$ to $\vec{y}$ does not meet the origin: $\sigma:[0,1] \rightarrow B_{*}^{n}(\overrightarrow{0}, R), \sigma(0)=\vec{x}, \sigma(1)=\vec{y}$. Then Arg $\circ \mathbf{g} \circ \sigma:[0,1] \rightarrow\left(-\frac{\pi}{2}, \frac{\pi}{2}\right)$ is

well-defined and continuous, and by the Intermediate Value Theorem, there exist $2 k+1$ points $t_{0}=0<t_{1}<t_{2}<\ldots<t_{2 k}=1$ so that $\left|\operatorname{Arg}\left(\mathbf{g}\left(\sigma\left(t_{j}\right)\right)\right)-\operatorname{Arg}\left(\mathbf{g}\left(\sigma\left(t_{j-1}\right)\right)\right)\right|<\frac{\pi}{2 k}$, so by Case 3 .,

$$
\left|\mathbf{g}\left(\sigma\left(t_{j}\right)\right)-\mathbf{g}\left(\sigma\left(t_{j-1}\right)\right)\right| \leq C_{1}^{1 / k}\left\|\sigma\left(t_{j}\right)-\sigma\left(t_{j-1}\right)\right\|^{1 / k}<C_{1}^{1 / k}\|\vec{y}-\vec{x}\|^{1 / k} .
$$

Then (22) follows, with $C_{2}=2 k C_{1}^{1 / k}$ :

$$
|\mathbf{g}(\vec{y})-\mathbf{g}(\vec{x})|=\left|\sum_{j=1}^{2 k} \mathbf{g}\left(\sigma\left(t_{j}\right)\right)-\mathbf{g}\left(\sigma\left(t_{j-1}\right)\right)\right| \leq \sum_{j=1}^{2 k}\left|\mathbf{g}\left(\sigma\left(t_{j}\right)\right)-\mathbf{g}\left(\sigma\left(t_{j-1}\right)\right)\right|<2 k C_{1}^{1 / k}\|\vec{y}-\vec{x}\|^{1 / k} .
$$

The above four Cases show that for $k>1$, (22) is satisfied for any $\vec{x}, \vec{y}$ by choosing the maximum constant $C_{2}=2 k C_{1}^{1 / k}$.

\section{Polynomial examples}

As mentioned in the Introduction, the $n=3, q=2$ case of an isolated zero of $\mathbf{f}$ can be viewed as an isolated point $\vec{p}$ in the intersection of two real surfaces $V\left(f_{1}\right) \cap V\left(f_{2}\right)$ in $\mathbb{R}^{3}$, which generically would meet in a space curve. In applications of geometry, it may be of interest to define a space curve implicitly by two polynomials, and then to remove any isolated points, which can be done by a continuous, semialgebraic homotopy as in Theorem 4.1, or by a homotopy as in Theorem 5.1 which is real analytic for $(\vec{x}, t)$ near but not equal to $(\vec{p}, 0)$.

Example 6.1. For $n=3, q=2$, where $\pi_{2}\left(S^{1}\right) \cong\{0\}$, consider the following pair of polynomials in $\mathbb{R}^{3}$, so that the varieties $V\left(f_{1}\right)$ and $V\left(f_{2}\right)$ meet only at $\overrightarrow{0}$ :

$$
\begin{aligned}
& f_{1}(x, y, z)=8 x^{2}+8 y^{2}-z^{2} \quad \text { Cone } \\
& f_{2}(x, y, z)=z\left(x^{2}+y^{2}\right)-x^{3} \quad \text { Cartan Umbrella }
\end{aligned}
$$

Corollary 3.8, applied to $\mathbf{f}=\left(f_{1}, f_{2}\right)$, shows that there exists some semialgebraic $\mathbf{g}$ close to $\mathbf{f}$ so that $V(\mathbf{g})=\varnothing$. However, in this case, it is not possible to construct $\mathbf{g}$ by merely translating the varieties:

$$
V\left(f_{1}\left(\vec{x}-\vec{\tau}_{1}\right), f_{2}\left(\vec{x}-\vec{\tau}_{2}\right)\right) \neq \varnothing,
$$

nor by choosing other level sets:

$$
V\left(f_{1}+C_{1}, f_{2}+C_{2}\right) \neq \varnothing
$$


for any constants $C_{1}, C_{2}$. Theorem 4.1 also applies, to show that there exists a continuous, semialgebraic homotopy that removes the isolated point of intersection, and analogously, Theorem 5.1 also applies. For this example, there is such a homotopy given globally by polynomials: $F=\left(F_{1}, F_{2}\right): \mathbb{R}^{3} \times \mathbb{R}^{1} \rightarrow \mathbb{R}^{2}:$

$$
\begin{aligned}
& F_{1}(x, y, z, t)=8 x^{2}+8 y^{2}-z^{2}+t^{2} \\
& F_{2}(x, y, z, t)=z\left(x^{2}+y^{2}\right)+z t^{2}-x^{3} .
\end{aligned}
$$

Example 6.2. For $n=4, q=3, \pi_{3}\left(S^{2}\right) \approx\{0\}$. A map not homotopic to a constant is given by the restriction of this polynomial map $\mathbb{C}^{2}=\mathbb{R}^{4} \rightarrow \mathbb{R}^{3}$ :

$$
\mathbf{f}\left(z_{1}, z_{2}\right)=\left(2 z_{1} \bar{z}_{2},\left|z_{1}\right|^{2}-\left|z_{2}\right|^{2}\right)
$$

to the unit sphere $S^{3}(\overrightarrow{0}, 1)=\left\{\left|z_{1}\right|^{2}+\left|z_{2}\right|^{2}=1\right\}$. The restriction is the Hopf map $\left(\mathrm{C}_{1}\right.$ Example 4.6; $\mathrm{C}_{2} \oint 8.2$ ), and this map $\mathbf{f}$ satisfies $V(\mathbf{f})=\{\overrightarrow{0}\}$. The homogeneous map $\mathbf{f}$ induces the Hopf map on any sphere, and so for any sphere $S^{3}(\overrightarrow{0}, R)$, there is an $\epsilon>0$, such that there does not exist even a continuous $\mathbf{g}$, nowhere zero inside the sphere and $\epsilon$-close to $\mathbf{f}$ on $S^{3}(\overrightarrow{0}, R)$.

Remark 6.3. The Proofs in Section 3 were not constructive, in that the notion of locally inessential merely asserts the existence of a homotopy, for example $\varphi$ in (4), and then we appealed to the Weierstrass Approximation Theorem to get $\mathbf{h}$ in (6). It should be noted that finding polynomial representatives of homotopy classes is a difficult problem with a long history, see $[\mathrm{B}], \mathrm{W}]$. In fact, one of the questions raised by [B] is on the existence of polynomial maps with isolated zeros.

\section{REFERENCES}

[Aikawa] H. AıkAwA, Hölder continuity of the Dirichlet solution for a general domain, Bull. London Math. Soc. (6) 34 (2002), 691-702. MR 1924196 (2003f:31007).

[A 1 D. Anker, On Removing Isolated Zeroes of Vector Fields by Perturbation, Ph.D. Thesis, University of Michigan, 1981. MR 2631252.

$\left[\mathrm{A}_{2}\right] \quad$ D. Anker, On removing isolated zeroes of vector fields by perturbation, Nonlinear Analysis, Theory, Methods, \& Applications (9) 8 (1984), 1005-1112. MR 0760200 (86d:58060).

[B] P. BAum, Quadratic maps and stable homotopy groups of spheres, Illinois J. Math. 11 (1967), 586595. MR 0220285 (36 \#3351).

[BCR] J. Bochnak, M. Coste, and M. Roy, Real Algebraic Geometry, MSM 36, Springer, 1998. MR 1659509 (2000a:14067).

[BFGJ] R. Brown, M. Furi, L. Górniewicz, and B. Jiang, eds., Handbook of Topological Fixed Point Theory, Springer, 2005. MR 2170491 (2006e:55001).

$\left[\mathrm{C}_{1}\right] \quad$ A. Coffman, $C R$ singular immersions of complex projective spaces, Beiträge zur Algebra und Geometrie (2) 43 (2002), 451-477. MR 1957752 (2003k:32052).

$\left[\mathrm{C}_{2}\right] \quad$ A. Coffman, Real congruence of complex matrix pencils and complex projections of real Veronese varieties, Linear Algebra Appl. 370 (2003), 41-83. MR 1994320 (2004f:14026).

$\left[D_{1}\right] \quad$ E. N. DANCER, On the existence of zeros of perturbed operators, Nonlinear Anal. (7) 7 (1983), 717-727. MR 0707080 (84m:47077).

$\left[\mathrm{D}_{2}\right] \quad$ E. N. DANCER, Bifurcation under continuous groups of symmetries, Systems of Nonlinear Partial Differential Equations (Oxford, 1982), 343-350, NATO ASI Ser. C 111, Reidel, Dordrecht, 1983. MR0725531 (85f:58025).

[D $\left.\mathrm{D}_{3}\right] \quad$ E. N. DAncer, Perturbation of zeros in the presence of symmetries, J. Austral. Math. Soc. Ser. A (1) 36 (1984), 106-125. MR 0720004 (85g:58027).

[Deloup] F. Deloup, The fundamental group of the circle is trivial, The American Mathematical Monthly (5) 112 (2005), 417-425. MR 2139574 (2005k:57002).

[E] A. Elgind, A topological obstruction to the removal of a degenerate complex tangent and some related homotopy and homology groups, Internat. J. Math. (5) 26 (2015), 1550025, 16 pp. MR 3345506 
[F] M. Fenille, Epsilon Nielsen coincidence theory, Cent. Eur. J. Math. (9) 12 (2014), 1337-1348. MR 3201326.

[G] P. Garabedian, Partial Differential Equations, Wiley, 1964. MR 0162045 (28 \#5247).

[GT] D. Gilbarg and N. Trudinger, Elliptic Partial Differential Equations of Second Order, Springer CIM, 2001. MR 1814364 (2001k:35004).

[LU] O. A. Ladyzhenskaya and N. N. URAL'Tseva, Linear and Quasilinear Elliptic Equations, translated from the 1964 Russian, Academic Press, 1968. MR 0244627 (39 \#5941).

[L] J. LeE, Introduction to Smooth Manifolds, Second ed., GTM 218, Springer, 2013. MR 2954043.

[M] V. G. MAZ'YA, Notes on Hölder regularity of a boundary point with respect to an elliptic operator of second order, J. Math. Sci. (N.Y.) (4) 196 (2014), 572-577; translated from Problemy Matematicheskogo Analiza 74 (2013), 117-121. MR 3391313.

[NNPV] M. Nestler, I. Nitschke, S. Praetorius, and A. Voigt, Orientational order on surfaces: the coupling of topology, geometry, and dynamics, J. Nonlinear Sci. (1) 28 (2018), 147-191. MR 3742799.

[PP] J. PALIS and C. Pugh, Fifty problems in dynamical systems, in Dynamical Systems - Warwick 1974, 345-353, LNM 468, Springer, 1975. MR 0391172 (52 \#11994).

[ST] C. Simon and C. Titus, Removing index-zero singularities with $C^{1}$-small perturbations, in Dynamical Systems - Warwick 1974, 278-286, LNM 468, Springer, 1975. MR 0650643 (58 \#31254).

[S] E. Spanier, Algebraic Topology, McGraw-Hill, 1966. MR 0210112 (35 \#1007).

[W] R. Wood, Polynomial maps from spheres to spheres, Invent. Math. 5 (1968), 163-168. MR 0227999 (37 \#3583).

Adam Coffman, Department of Mathematical Sciences, Purdue University Fort Wayne, Fort WAYNE, IN 46805, USA

E-mail address: CoffmanA@pfw.edu

Jiří Lebl, Department of Mathematics, Oklahoma State University, Stillwater, OK 74078, USA

E-mail address: lebl@math.okstate.edu 